\title{
DURABILIDAD NATURAL DE LA MADERA DE DOS ESPECIES APTAS PARA LA INDUSTRIA DE LA CONSTRUCCIÓN
}

\author{
Elisa Alicia Bobadilla*, Obdulio Pereyra *, Fidelina Silva*, Alicia Mónica Stehr** \\ *Ing. Forestal, M.Sc., Facultad de Ciencias Forestales, UNaM - elisa@facfor.unam.edu.ar - opereyra@facfor.unam.edu.ar - \\ fidelina@facfor.unam.edu.ar \\ **Ing. Forestal, Facultad de Ciencias Forestales, UNaM - astehr@facfor.unam.edu.ar \\ Recebido para publicação: 15/06/2005 - Aceito para publicação: 03/10/2005
}

\begin{abstract}
Resumen
Durabilidad natural de la madera de dos especies aptas para la industria de la construcción. El objetivo de este trabajo fue determinar la resistencia natural de la madera de Pawlonia tomentosa y Melia azedarach, frente al ataque de tres cepas de los hongos xilófagos Picnoporus sanguineus, Laetiporus sulfureus y Ganoderma applanatum, bajo dos condiciones: no tratadas y después de sufrir ciclos de lavado. Los objetivos específicos fueron a) constatar si la resistencia de las maderas estudiadas es alterada, por el ataque de los hongos después de la eliminación de las sustancias solubles en agua contenidas en la madera, y b) clasificar en categorías de resistencia de acuerdo al porcentaje de pérdida de peso siguiendo los Criterios de Findlay. Se utilizaron 5 probetas de maderas de $3 \times 1 \times 0,5 \mathrm{~cm}$, por especie y por hongo, las mismas fueron sometidas en laboratorio a la acción de los hongos y colocadas en estufa a $28^{\circ} \mathrm{C}$ durante tres meses. Transcurrido este periodo, se pesó cada una de las probetas y se determinó el porcentaje de pérdida de peso con respecto a su valor inicial. La madera de Pawlonia resultó moderadamente durable a la acción de los hongos Picnoporus y Laetiporus, independientemente de los tratamientos, resultando no durable al ataque del hongo Ganoderma. La madera de Melia resultó ser no durable y perecedera frente a la acción de las tres especies de hongos.
\end{abstract}

Palabras-clave: Resistencia natural; hongos xilófagos; Pawlonia tomentosa; Melia azedarach.

\begin{abstract}
Natural durability of wood of two species apt for contruction industries. The aim of this work was to determine the natural resistance of Pawlonia tomentosa and Melia azedarach, woods against Picnoporus sanguineus, Laetiporus sulphureus and Ganoderma applanatum decay fungi, under two conditions: natural non treated wood and after suffering cycles of washing. The specific aims were, a) to established $\mathrm{e}$ if the natural resistance of the wood is altered, by the fungi studied after the removal of the soluble water substances b) to classify the wood resistance according to Findlay's Criteria by the loss of weight due to fungi decay. Five probes $3 \times 1 \times 0.5 \mathrm{~cm}$ were used for each treatment. The treatment were factorial of $2 \times 2 \times 3$ being 2 kinds of woods, 2 different treatment and 3 species of fungi. The probes were kept at $28{ }^{\circ} \mathrm{C}$ in darkness during a 3 month period. The probe were weighted at the beginning and end of the experiment. The difference of weight was expressed as percentage of the initial value. The wood of Pawlonia was moderately durable independently of the treatments, to the action of the Picnoporus fungus, and non durable to Laetiporus and Ganoderma fungi. Melia wood was not durable and perishable, under the action of the three fungi species.

Keywords: Natural resistance; decay fungi; Pawlonia tomentosa; Melia azedarach.
\end{abstract}

\section{INTRODUCCIÓN}

El uso de la madera, para un fin determinado depende principalmente de sus propiedades físicas, mecánicas y de su carácter estético. Es necesario sin embargo no dejar de considerar que estas propiedades son modificadas, en mayor o menor escala, por las alteraciones que sufre la madera a lo largo del tiempo. 
Entre las causas que provocan alteraciones de la madera, ocupan un lugar muy importante los agentes biológicos, los cuales pueden ser agrupados en tres categorías: hongos xilófagos, insectos y animales marinos.

Los hongos xilófagos son responsables de grandes pérdidas económicas por la destrucción de enormes cantidades de madera, principalmente aquellas destinadas a la construcción de viviendas. Tal es la importancia, que ha dado origen a la industria de la preservación de madera.

Se entiende por durabilidad natural al conjunto de propiedades de la madera, características de cada especie, que le confieren una determinada durabilidad en servicio, cuando son utilizadas sin ningún tratamiento preservativo.

Los objetivos de este trabajo fueron determinar la resistencia natural de la madera de las especies kiri (Pawlonia tomentosa (Thumb ex Murr) Steud.) y paraíso (Melia azedarach L.), expuestas al ataque de tres cepas de hongos xilófagos, bajo dos condiciones: natural y luego de sufrir ciclos de lavados y clasificar las maderas de las especies estudiadas de acuerdo a los Criterios de Findlay en categorías de resistencias.

\section{REVISIÓN BIBLIOGRÁFICA}

Ante la escasez de maderas del bosque nativo y un aumento permanente en los requerimientos por parte de la industria, que demanda alternativas a la clásica madera de pino, y a efectos de suplir deficiencias en la obtención de materia prima y datos que son necesarios para el sector industrial, González et al (2003), han estudiado el comportamiento tecnológico de las maderas de cinco especies forestales que prosperan en la Provincia de Misiones. El presente estudio complementa el citado trabajo aportando información respecto a la durabilidad natural de las especies kiri y paraíso.

\section{Características generales de las especies estudiadas}

Melia azedarach L. - paraíso (Clase: Dicotiledóneas - Familia: Meliaceae): Su madera presenta rasgos parecidos al cedro misionero, con albura estrecha de color amarillo ocre, netamente diferenciada del duramen castaño rojizo, su densidad es de $0,520 \mathrm{~g} / \mathrm{cm}^{3}$. Las contracciones son medianas y la estabilidad dimensional intermedia, comportándose correctamente en servicio. Estaciona sin dificultad y relativamente rápido, admitiendo normas aceleradas de secado artificial. Es poco durable en contacto con el suelo. El duramen es poco penetrable, Tinto (1978), con absorción mediana. Es una madera blanda fácil de trabajar, se presta bien para proceso de rebobinado y corte plano, admite bien las colas, lustres y barnices. (Leonardis, 1977).

Paulownia tomentosa (Thumb ex Murr) Steud. - kiri (Clase: Dicotiledóneas-Familia: Bignoniaceae): Su madera es buena para determinados usos, tiene albura y duramen poco diferenciado, siendo éste último de color blanco-amarillento con tonalidades rosadas. Recibe con facilidad adhesivos y pinturas. La densidad media es de $0,240 \mathrm{~g} / \mathrm{cm}^{3}$, presentando buena estabilidad dimensional en servicio. Es poco durable a la intemperie. Puede impregnarse sin dificultad. Se trabaja con suma facilidad, resultando una madera blanda, dócil, sin tendencia a rajaduras en el clavado, pero con poca adherencia. Toma bien los tintes, barnices, pintura y colas. Se utiliza para embalajes, muebles de todo tipo, tacos de zapatos, persianas, entre otros (Leonardis, 1977).

\section{Durabilidad natural}

La durabilidad natural comprende aquellas características de resistencia que posee la madera sin tratamiento químico frente al ataque de hongos, insectos, perforadores marinos y otras influencias. Normalmente se mide, como el tiempo en años durante el cual una madera es capaz de mantener sus propiedades mecánicas estando puesta en servicio empotrada en contacto con el suelo o el agua. La mayoría de las maderas tienen una durabilidad diferente frente a los diversos organismos que la pueden degradar.

Entre las especies durables, la naturaleza y cantidad de los extractivos del tejido leñoso son muy variables. Algunas veces, la durabilidad es debida a la toxicidad para los hongos de los compuestos que se solubilizan en el alcohol-benceno, otras en cambio se deben a los solubles en agua caliente.

Las maderas pueden ser clasificadas según su uso y cada una de ellas, sujeta a distintos niveles de riesgo de ataque de hongos (García Sola, 1974): 1. Maderas en contacto directo y permanente con el 
suelo: postes de luz, durmientes. Riesgo importante y constante con amenaza de destrucción. 2. Madera que no está en contacto directo con el suelo, pero que puede sufrir períodos largos de exposición a la humedad: estructuras a la intemperie. Riesgo importante de ataque con lenta evolución. 3. Madera que no está en contacto directo con el suelo, pero que sufre rehumedificaciones por acción directa de lluvias: aleros de techos. Riesgo constante de evolución lenta. 4. Maderas que no están en contacto con el suelo y al abrigo de la intemperie. Sin riesgo de ataque. 5. Maderas totalmente sumergidas en agua salada que no sufren ataque alguno.

Ante la acción de agentes micóticos las distintas maderas presentan diferente resistencia. Esto se debe a su estructura y a las proporciones de sustancias químicas contenidas en los tejidos leñosos, variables según la especie, la edad del árbol y las condiciones de desarrollo (Carballeira López et al, 1986).

La durabilidad natural de las maderas se clasifica, en el caso de deterioro producido por hongos, en cinco clases, (Junta del acuerdo de Cartagena, 1988), las mismas son:

- Muy durable - más de 20 años de duración

- Durable - 15 a 20 años de duración

- Moderadamente durable - 15 a 10 años de duración

- Poco durable - 10 a 5 años de duración

- No durables - menos de 5 años de duración

\section{Hongos}

Los hongos pudridores de la madera pueden ser agrupados en dos categorías según el tipo de pudrición producida. Estos dos grupos son denominados hongos de pudrición blanca y hongos de pudrición castaña.

Los hongos de pudrición castaña remueven selectivamente celulosa y hemicelulosa de la madera. La madera podrida por estos hongos de pudrición castaña pierde rápidamente sus propiedades de solidez y experimentan roturas drásticas.

Los hongos de pudrición blanca tienen un sistema de enzimas celulosa y lignasa que le permiten degradar todos los componentes de las paredes celulares de la madera. Algunos, sin embargo, remueven lignina más rápidamente.

El contenido de humedad en la madera, que favorece el deterioro por estos organismos, es mayor al de punto de saturación de las fibras (p.s.f.), el valor oscila entre el $30 \%$ y el $50 \%$ de humedad. Por debajo del $20 \%$ su acción es prácticamente nula.

La temperatura óptima de desarrollo de los hongos oscila entre los $20^{\circ} \mathrm{C}$ y $30^{\circ} \mathrm{C}$, la actividad es prácticamente nula por debajo de los $4{ }^{\circ} \mathrm{C}$ y superior a los $40^{\circ} \mathrm{C}$.

El oxígeno es un elemento fundamental para el desarrollo de los hongos, el porcentaje debe ser siempre mayor al $20 \%$ de volumen de oxígeno por volumen de madera.

\section{Descripción general de las especies de hongos ensayadas}

Ganoderma applanatum: Es una especie leñosa no comestible, siendo parásita de muchos árboles en los que provoca una podredumbre blanca muy activa y cuando la planta muere es saprofita. Aparece en todas las épocas del año. Causa en árboles en pie una pudrición corrosiva y lo que se denomina corazón rojo y en las maderas aserradas el pasmo.

Laetiporus sulphureus: Vive como parásito en troncos de árboles frondosos, vivos o muertos, también sobre frutales, siendo muy raro el que se desarrolle sobre coníferas, puede ser encontrado desde primavera a otoño. Es comestible solamente en su zona periférica y cuando el ejemplar es muy joven. Produce pudrición castaña, parda o cúbica, teniendo la particularidad de extraer todo el contenido celular y luego destruir la celulosa y la hemicelulosa, dejando intacta la lignina. Produce una pudrición destructiva de tipo prismático con láminas de micelio intercaladas.

Pycnopurus sanguineus: Habita sobre troncos, ramas, tocones, empalizadas de maderas frondosas, pudiendo hacerlo también sobre coníferas. Como hongo saprófito no ataca árboles vivos sino madera muerta siendo su estructura miceliar, en el interior de la misma, de color rojo. Produce pudrición blanca, también llamada pudrición fibrosa, descompone la lignina y algo de celulosa y hemicelulosa. 


\section{Requerimientos en maderas para la construcción de viviendas}

El empleo de la madera en la vivienda asume características particulares, ya que los volúmenes y tipos utilizados, así como los de sus manufacturas, dependen de las propiedades de las especies seleccionadas y de los sistemas constructivos y modelos habitacionales proyectados.

Como todo material de construcción, la madera presenta ventajas e inconvenientes frente a las exigencias de trabajo en las condiciones de uso.

Las características positivas que acusa la madera son:

- Se trata de un material renovable.

- Es relativamente liviana, lo que permite manejarla y transportarla sin grandes esfuerzos.

- Puede trabajarse fácilmente, dándosele la forma requerida, sin necesidad de equipos complicados.

- Por absorber líquidos, permite tratarla con pinturas para su protección o decoración.

- Es mala conductora del calor, sonido y electricidad, por lo que resulta un material aislante excelente.

- Se contrae o dilata muy poco con los cambios de temperaturas.

- Absorbe choques y vibraciones mejor que otros materiales.

- La madera no cristaliza ni se vuelve quebradiza, como los metales ni pierde sus características de cohesión, como el hormigón, cuando se somete a temperaturas muy bajas.

- Antes de quebrarse presenta síntomas que lo anuncia, lo que resulta de suma importancia para prevenir accidentes.

- $\quad$ Puede curvarse mediante el calor, y mantener la nueva forma indefinidamente.

En contraposición a estas características comunes de las maderas, que significan una ventaja en su empleo, este material posee algunas limitaciones tecnológicas, tales como:

- La madera maciza debe ser unida con conectores metálicos, o encolada, para aumentar la longitud de las piezas.

- Tiene una dureza y resistencia limitadas.

- Como sustancia higroscópica, varia su contenido de humedad de acuerdo con los cambios ambientales, lo que origina cambios en la forma y dimensiones de las piezas.

- Es susceptible al ataque de hongos, que la manchan y desintegran, y a insectos que la perforan.

- Es combustible y, una vez encendida, desprende gases que tienden a aumentar la temperatura y con ello la combustión, aún hasta después que la fuente originaria de calor ha desaparecido.

- La solidez de la madera es variable, no sólo entre las especies, sino dentro de una misma especie.

Las desventajas de la madera pueden solucionarse con las siguientes medidas:

- Las deformaciones pueden aminorarse y evitarse mediante el correcto secado, aplicación de productos hidrófugos y utilización de elementos laminados y contrachapados.

- Muchas maderas son naturalmente resistentes a la acción de hongos e insectos. Aquellas susceptibles pueden ser protegidas eficazmente por medio de impregnación con productos químicos adecuados.

- Mediante, el empleo de maderas densas, de baja combustibilidad, escuadrías grandes y aplicación de sustancias ignífugas, se reduce sustancialmente el riesgo del fuego.

- Los sistemas de ensamblado-laminado permiten obtener piezas de madera con largos y espesores considerables, lográndose estructuras con luces amplias.

\section{Conservación de la madera para la construcción}

Desde el punto de vista profesional de la construcción, existen tres procedimientos de distinta índole que facilitan o permiten ofrecer a los componentes leñosos una prolongada vida útil.

El primero es el secado, reduce el contenido de humedad por debajo del $20 \%$, este método es el más simple y económico y cubre dos objetivos en la madera: evitar o disminuir los fenómenos físicos 
anormales de forma y estructura que conspiran contra su aplicación, como así también el ataque de los hongos xilófagos e insectos que afectan al leño.

Si el 20\% de humedad no se altera, cualquier madera resulta inmune al ataque de los hongos e insectos minimizando los movimientos perjudiciales.

Cuando no es factible mantener aquel umbral de humedad o resulta riesgoso asegurarlo, máxime si la madera va estar en contacto con el exterior, suelo o intemperie, o será utilizada en saunas, sótanos, etc., debe recurrirse al segundo método de protección denominado preservación.

El tercer método se conoce como conservación por diseño, se trata de medidas que se implementan y se insertan dentro del arte de proyectar y construir con piezas leñosas.

\section{Método para determinar la durabilidad de la madera}

La durabilidad de las maderas se determina, a través de métodos de laboratorio y de campo. Aunque las técnicas son muy variadas, los métodos más generalizados son los que se realizan en laboratorios, se los conoce como "Pruebas Aceleradas" por su corta duración. Los que se llevan a cabo en el campo o "Cementerios", que son pruebas definitivas de durabilidad, tienen el inconveniente de demandar mayor tiempo.

Los datos que se obtienen en laboratorio se denominan "Índices" debiendo la información ser comparada con la que se logra en los cementerios para que sea más confiable.

\section{Pruebas Aceleradas en Laboratorio}

Las pruebas para la determinación de la durabilidad o resistencia natural de una madera en laboratorio permiten obtener información sobre la efectividad de un producto preservante o el desempeño de una cepa de hongos en un tiempo relativamente corto, de 3 a 6 meses.

Estos ensayos demandan:

1. Control estricto de las técnicas y procedimientos de laboratorio para establecer el trabajo en condiciones de asepsia.

2. Control de las variables más importantes que intervienen en el proceso, como ser, temperatura, humedad relativa, ventilación.

3. Simplificación del objeto de estudio, poco material, en este caso un número pequeño de probetas y cepas de algunos hongos.

Para la realización de ensayos de laboratorio se conocen varias metodologías, para este proyecto se siguió la técnica sugerida por la Norma IRAM 9518 (Instituto Argentino de Racionalización de Materiales, 1962), que consiste en desarrollar las cepas de hongos en un medio de cultivo compuesto por agar-agar y extracto de malta, colocándose sobre el mismo probetas por un lapso de tres meses.

El dato final de este ensayo es el porcentaje de pérdida de peso después del ataque de los hongos respecto del inicio del mismo. Con esta información podemos clasificar a las maderas empleando distintos tipos de tablas, en este estudio se utilizó el "Criterio de Findlay", la cual es presentada en la tabla 1, que no ofrece diferencias significativas respecto a las otras (FINDLAY W.P.K., 1951).

Tabla 1. Clasificación por resistencia de las maderas según pérdida de peso.

Table 1. Clasification of wood resistence by weight lost.

\begin{tabular}{cc}
\hline Pérdida de peso & Categoría de Resistencia \\
\hline Inferior al $5 \%$ & Muy Resistente \\
$5 \%$ al $10 \%$ & Resistente \\
$10 \%$ al $20 \%$ & Moderadamente resistente \\
$20 \%$ al $30 \%$ & No resistente \\
Superior al $30 \%$ & Sin resistencia \\
\hline
\end{tabular}

Es muy importante tener en cuenta que los resultados aportados por ensayos de laboratorio pueden no corresponder, si las condiciones en cuanto a ambiente o constructivas no son similares a las del ensayo. 


\section{MATERIALES Y MÉTODOS}

\section{Madera}

Fueron utilizadas maderas de las especies Melia azederach (paraíso) y Pawlonia tomentosa (kiri), provenientes de industrias madereras locales, el material utilizado fue seleccionado totalmente al azar teniendo presente que cumpla con la característica de ser sin nudos, manchas ni pudriciones.

\section{Hongos}

Se ensayaron tres cepas de hongos que fueron provistas por el Departamento de Ciencias Biológicas de la Facultad de Ciencias Exactas y Naturales de la Universidad de Buenos Aires, (UBA), a saber: Laetiporus sulphureus; Pycnoporus sanguineus y Ganoderma applanatum .

\section{Metodología}

Se sigue la metodología sugerida por las Normas IRAM No 9518 (Instituto Argentino de Racionalización de Materiales,1962), "Toxicidad, permanencia y eficacia de preservadores de maderas".

\section{Diseño estadístico}

Como diseño experimental se adoptó el totalmente aleatorizado, con análisis factorial 2 por 3 por 2, siendo dos las condiciones iniciales de las probetas de madera, (Natural y Lavadas); tres las especies de hongos y dos las diferentes especies de madera. La variable medida fue el peso de las probetas al inicio y al final del experimento (Area, 1996).

\section{Etapas}

Preparación de las probetas

Para los ensayos se utilizaron 60 probetas de $3 \times 1 \times 0,5 \mathrm{~cm} .30$ probetas por especie, 15 de las cuales se emplearon en condiciones naturales y las 15 restantes sometidas a lavados en extractor Sohxlet.

\section{Estabilización de las probetas}

Las probetas fueron colocadas a ambiente de laboratorio durante 72 horas. hasta que las mismas alcanzaran un peso estable, las mediciones fueron repetidas tres veces, como mínimo, siendo esta primera variable medida denominada: Peso Estabilizado $1\left(\mathrm{P}_{\mathrm{est} 1}\right)$.

\section{Humectación y esterilización de las probetas de maderas naturales}

Previo a la colocación de las probetas en los tubos de ensayo con cultivos de hongos fueron separadas 15 probetas destinadas a valorar la resistencia natural, las cuales fueron colocadas en un soporte en el interior de un desecador con agua destilada en el fondo, simulando un ambiente semejante al de una cámara húmeda.

Transcurridas 48 horas las probetas fueron colocadas en estufa a una temperatura de $110{ }^{\circ} \mathrm{C}$ durante $1 / 2$ hora, con el fin de esterilizarlas. Una vez cumplida esta etapa, y sin extraer las probetas de la estufa se bajó la temperatura a $30{ }^{\circ} \mathrm{C}$ manteniéndola en esa condición por 12 horas, de modo que las probetas alcanzaran una humedad constante de aproximadamente $30 \%$.

\section{Esterilización y humectación de las probetas sometidas a ciclos de lavado}

Una vez separadas por especies las 15 probetas restantes fueron colocadas en un extractor Sohxlet cargado con agua destilada, efectuándose diez (10) pasadas de líquido a través de la cámara. El objetivo de este procedimiento fue simular un proceso de exposición de los cuerpos de prueba a la intemperie, se presupone que es el número necesario de lavadas para que todas las sustancias solubles en agua fueran extraídas durante esta operación. Finalizado este proceso, y para garantizar la esterilidad, el agua de la cámara fue hervida aproximando la llama del mechero, tratando de que no se pierda vapor por la parte abierta del Sohxlet, tras lo cual se considera que las probetas tienen suficiente humedad, lo que puede estimarse entre $35 \%$ y $40 \%$. 
Determinación de la pérdida de peso de las probetas sometidas a ciclos de lavado

El objetivo de esta medición fue determinar la pérdida de peso cuando son extraídas las sustancias solubles durante el lavado en Sohxlet. Por cada especie se llevaron tres probetas de las mismas dimensiones a estufa a $110{ }^{\circ} \mathrm{C}$ hasta alcanzar estado anhidro, midiéndose posteriormente su peso, denominado "peso anhidro antes del lavado" $\left(P_{a} l\right)$. Posteriormente las probetas fueron dejadas en ambiente de laboratorio durante 72 horas y luego colocadas en el extractor Sohxlet. Se hizo circular por la cámara 10 pasadas de agua destilada para proceder luego a retirarlas, siendo estas escurridas y llevadas nuevamente a estufa a la misma temperatura que en el paso anterior hasta recuperar su estado anhidro, denominado a partir de este momento "peso anhidro después del lavado" $\left(P_{d l}\right)$. Con estos datos se procedió a calcular el porcentaje de pérdida de peso por lavado (\% PL), utilizando la siguiente expresión: $\% P L=\frac{P_{a l}-P_{d l}}{P_{a l}} \times 100 ;$ que será descontado de las probetas lavadas, utilizando un porcentaje promedio por especie.

\section{Preparación del medio de cultivo}

Como medio de cultivo de las cepas, se empleó el indicado en la Norma IRAM No 9518 (Instituto Argentino de Racionalización de Materiales, 1962), apartado G/16, donde el medio está compuesto por $25 \mathrm{~g}$ de agar-agar y $15 \mathrm{~g}$ de extracto de malta, por cada $1000 \mathrm{~g}$ de agua destilada. Esta solución fue distribuida en tubos de ensayo marca Pirex de $200 \mathrm{~mm}$ de largo y $20 \mathrm{~mm}$ de diámetro, obturados con tapón de algodón. Los tubos de ensayo fueron llevados a autoclave para ser esterilizados a 1,5 atmósferas de presión por el término de 30 minutos; siendo posteriormente colocados en grillas de forma levemente inclinada para que al solidificarse el medio se obtenga un pico de flauta suficientemente extenso.

\section{Selección y cultivo de hongos}

Se utilizaron las cepas de tres hongos por ser las más comunes en nuestra zona: Pycnoporus sanguineus, Ganoderma applanatum. y Laetiporus sulphureus

Trozos de micelio de las cepas de los hongos fueron colocados dentro de los tubos de ensayo con el medio preparado para el desarrollo de los mismos teniendo en cuenta todos los cuidados de asepsia por medio de la utilización de una cámara de flujo laminar. Una vez finalizada esta etapa los mismos fueron colocados en una estufa de cultivo a una temperatura entre $26^{\circ} \mathrm{C}$ y $28^{\circ} \mathrm{C}$ y una humedad relativa de $30 \%$, durante 14 días, tiempo suficiente para el desarrollo vigoroso del micelio que cubría totalmente la superficie formada por el pico de flauta.

\section{Colocación de las probetas en tubos con micelio de hongos}

Las probetas de madera, esterilizadas y humectadas, fueron colocadas asépticamente, sobre el micelio desarrollado en los tubos, como lo muestra la Figura 1.

Cada tubo fue identificado con una letra representando la especie, el número de la probeta, la especie de hongo y el tratamiento (natural o lavada).

Una vez colocadas las probetas en los tubos, los mismos fueron dispuestos horizontalmente en estufa de cultivo a $27 \pm 1{ }^{\circ} \mathrm{C}$ y una humedad relativa de $30 \%$ por el transcurso de tres meses.

\section{Retiro de las probetas y estabilización}

Con el fin de verificar el desarrollo uniforme del micelio y la posibilidad de contaminación, las probetas fueron examinadas semanalmente. Al cabo de tres meses fueron retiradas las probetas de cada tubo de ensayo siendo limpiadas con un paño para eliminar todo vestigio de micelio de hongos evitándose así que siga actuando una vez retiradas de la estufa de cultivo. Las probetas permanecieron en ambiente de laboratorio en esas condiciones por un período de 72 horas, tiempo en el que se completó la limpieza.

Posteriormente las probetas fueron llevadas a cámara húmeda durante un tiempo, que en ninguno de los casos fue inferior a 48 horas, para lograr de esta manera alcanzar la misma humedad de estabilización del inicio del ensayo, un rango entre 30\% y 40\%. Se controló el peso hasta que éste permaneciera constante. Este dato de peso de cada probeta, después de ser sometidas a la acción de los hongos, fue tomado como "peso estabilizado 2 " $\left(P_{e t 2}\right)$. 


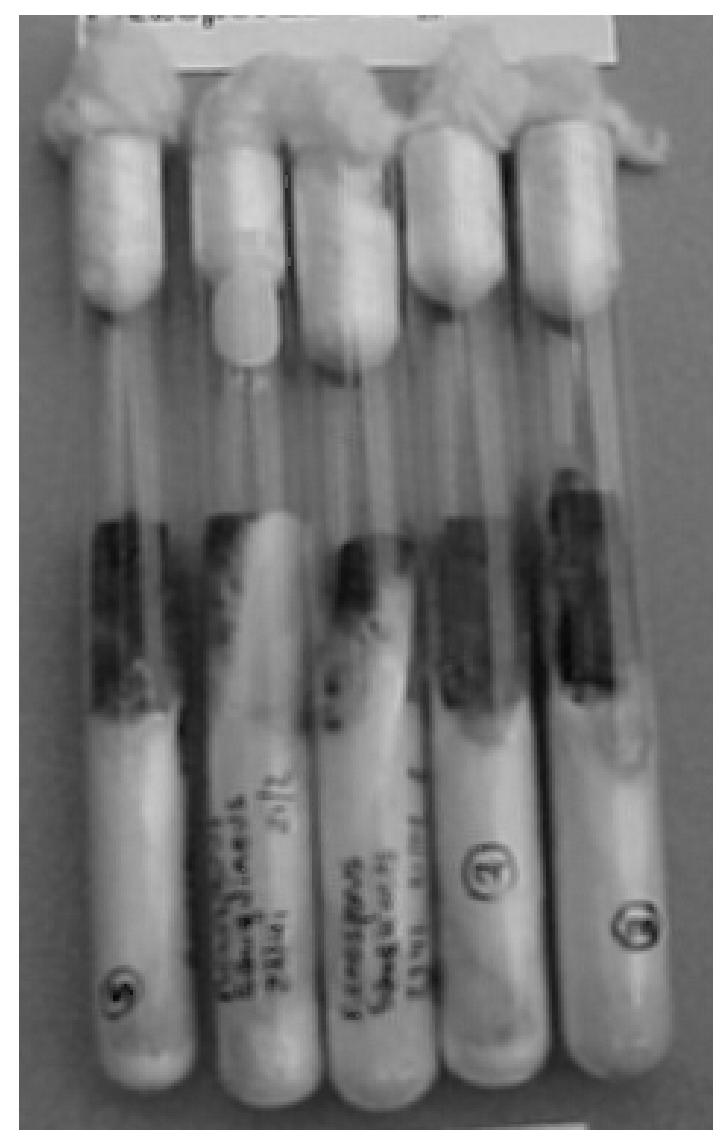

Figura 1. Tubos cultivados a ser ubicados en estufa.

Figure 1. Cultivated tubes to be placed in stove.

\section{Pérdida de peso de las probetas}

Siendo la humedad de estabilización para cada tipo de madera la misma antes y después del ataque de los hongos, la pérdida de peso en tejido leñoso $(P T L)$ se calcula con la siguiente expresión:

$$
\triangle P T L \%=\frac{P_{\text {est } 1}-P_{\text {et } 2}}{P_{\text {est } 1}} \times 100
$$

Aplicando esta expresión tendremos dos resultados según estemos hablando de:

a. Madera natural: nos dará el porcentaje de pérdida de tejido leñoso por la acción del hongo referida al peso de tejido inicial.

b. Madera lavada: como el peso estabilizado $1\left(P_{\text {est } t}\right)$ es el peso sin lavado antes del ataque, y el peso estabilizado $2\left(P_{\text {et }} 2\right)$ es el peso después de lavado y del ataque hongo; la expresión indica pérdida de peso de tejido leñoso por la acción conjunta del lavado y del hongo, referida al peso de tejido leñoso inicial. Por lo tanto al valor obtenido con la expresión anterior debemos restarle el porcentaje de pérdida de peso por lavado (\% P L), obtenido por el método descrito con anterioridad, la diferencia $\triangle P T L \%--\% P L$, será igual a la pérdida porcentual de peso de la madera lavada por acción del hongo referida al peso de tejido leñoso inicial. 


\section{RESULTADOS}

\section{Pérdida de peso de las especies de madera}

En la tabla 2 se presenta el promedio de pérdida de peso en porcentaje, desvío estándar y coeficiente de variación por tratamiento y por hongos.

Tabla 2. Porcentaje de Pérdida de Peso según tratamiento, especie maderera y hongo.

Table 2. Lost weight percentaqe by treatment, wood specie and fungi.

\begin{tabular}{lcccccccc}
\hline Especie & \multicolumn{5}{c}{ Lavado } & \multicolumn{4}{c}{ Natural } \\
\hline \multirow{2}{*}{ Paraíso } & Hongos & Media & Desv. Est. & CV & Hongos & Media Desv. Est. & CV \\
& Laetiporus & 56,21 & 2,11 & 3,74 & Laetiporus & 49,57 & 4,07 & 8,21 \\
& Picnoporus & 35,49 & 3,88 & 10,94 & Picnoporus & 33,18 & 3,37 & 10,16 \\
& Ganoderma & 28,61 & 1,62 & 5,68 & Ganoderma & 25,72 & 2,75 & 10,70 \\
\hline kiri & Ganoderma & 33,20 & 4,58 & 13,80 & Ganoderma & 29,51 & 3,73 & 12,63 \\
& Laetiporus & 15,13 & 2,82 & 18,63 & Picnoporus & 10,94 & 1,10 & 10,01 \\
& Picnoporus & 14,75 & 1,96 & 13,28 & Laetiporus & 9,92 & 1,51 & 15,18 \\
\hline
\end{tabular}

De la misma se puede extraer los siguientes resultados:

a. En el paraíso se mantuvo el mismo orden descendente de porcentaje de pérdida en ambos tratamientos y para cada tipo de hongo en estudio. Los pares de valores de porcentaje de pérdida (lavada y natural) fueron muy similares. Los hongos más agresivos para el paraíso fueron Laetiporus sulphureus y Picnoporus sanguineus, donde el porcentaje de pérdida de peso supera el $30 \%$ y la madera, según la criterio de Findlay es una madera perecedera. La madera frente a la acción del hongo Ganoderma applanatum es una madera no durable.

b. La madera de kiri fue más susceptible al ataque del hongo Ganoderma applanatum, para la madera natural y después de sufrir ciclos de lavado, los porcentajes de pérdidas son similares, cercanos al 30\%, lo que la hace una madera no durable. Para los hongos Laetiporus sulphureus y Picnopurus sanguineus e independientemente de las condiciones iniciales de ensayo, las pérdidas de peso fueron entre $10 \%$ y $15 \%$, por lo que la madera según el criterio de Findlay es moderadamente durable.

La tabla 3 muestra que las diferencias son significativas debido a los tratamientos y no al azar. En este trabajo es importante resaltar este hecho debido a que, dentro de las características requeridas, la muestra de madera de la cual provienen las probetas utilizadas, es de origen comercial y seleccionada al azar.

Tabla 3. Análisis de variancia para la interacción tratamiento-hongo-madera.

Table 3. Variance análisis for treatment-fungi-wood interaction.

\begin{tabular}{lccccc}
\hline $\begin{array}{l}\text { Fuente de } \\
\text { variación }\end{array}$ & Suma de cuadrados & g. de $\mathbf{l}$. & Cuadrado medio & F & P \\
\hline Efecto & 60396,0508 & 1 & 60396,0508 & 5789,72412 & 0 \\
Error & 1251,79126 & 120 & 10,4315939 & & \\
\hline
\end{tabular}

\section{Clasificación de Findlay}

En base a la información presentada previamente, y de acuerdo al Criterio de Findlay presentada en la tabla 1, se clasifica a las maderas en grados de resistencia de acuerdo a la pérdida de peso, tratamiento recibido y hongo (Tabla 4).

\section{CONCLUSIONES}

Los resultados obtenidos en este trabajo permiten arribar a las siguientes conclusiones:

- Independientemente de los tratamientos recibidos la madera de Paraíso es perecedera frente a la acción de los hongos Picnoporus sanguineus y Laetiporus sulphureus y no durable en caso de Ganoderma applanatum. 
- La madera de kiri frente a la acción del hongo Laetiporus sulphureus, cuando la misma es sometida a ciclos de lavado es no durable, mientras que para el mismo hongo en estado natural es moderadamente durable.

- La madera de kiri es moderadamente durable, bajo la acción del hongo Picnoporus sanguineus, para ambas condiciones de trabajo.

- La madera de kiri que es sometida a ciclos de lavado es perecedera frente a la acción del hongo Ganoderma applanatum y no durable para la madera natural.

- De acuerdo a los resultados antes expuestos, las maderas de Paraíso y kiri solo son aptas para la industria de la construcción, si se toman recaudos en los diseños de protección para evitar contacto directo con los agentes ambientales que favorecen el desarrollo de los agentes xilófagos o utilizando preservantes químicos para la madera.

Tabla 4. Clasificación de la madera en grados de resistencias según ataque de hongos.

Table 4. Wood clasification in degrees of resistance according to attack of fungi.

\begin{tabular}{lcccc}
\hline \multirow{2}{*}{ Hongo } & \multicolumn{3}{c}{ Paraíso } & \multicolumn{2}{c}{ Kiri } \\
\cline { 2 - 5 } & Lavada & Natural & Lavada & Natural \\
\hline Laetiporus sulphureus & $\begin{array}{c}\text { Perecedera } \\
(56,2)\end{array}$ & $\begin{array}{c}\text { Perecedera } \\
(49,6)\end{array}$ & $\begin{array}{c}\text { Moderadamente } \\
\text { Durable } \\
(15,3)\end{array}$ & $\begin{array}{c}\text { Durable } \\
(9,9)\end{array}$ \\
\hline Picnoporus sanguineus & $\begin{array}{c}\text { Perecedera } \\
(35,5)\end{array}$ & $\begin{array}{c}\text { Perecedera } \\
(33,2)\end{array}$ & $\begin{array}{c}\text { Moderadamente } \\
\text { Durable } \\
(14,8)\end{array}$ & $\begin{array}{c}\text { Moderadamente } \\
\text { Durable } \\
(10,4)\end{array}$ \\
\hline Ganoderma applanatum & $\begin{array}{c}\text { No Durable } \\
(28,6)\end{array}$ & No Durable $(25,7)$ & $\begin{array}{c}\text { Perecedera } \\
(33,2)\end{array}$ & No Durable $(29,5)$ \\
\hline * Los valores entre paréntesis expresan la pérdida de peso de las muestras en porcentaje $(\%)$. &
\end{tabular}

* Los valores entre paréntesis expresan la pérdida de peso de las muestras en porcentaje (\%).

\section{REFERENCIAS}

AREA, M. C. Apuntes Curso Metodología de la Investigación: diseños experimentales. [S.1.]: UNAM/ Facultad de Ciencias Exactas Químicas y Naturales, 1996.

CARBALleIRA LÓPEZ; S.; MILANO, G. Manual de preservación de maderas. San Pablo: Instituto de Pesquisas Tecnológicas do Estado de San Pablo, 1986. v. 2, cap. 10.

FINDLAY, W. P. K. The value of laboratory test on wood preservatives. [S.1.]: Convention British Wood Preserving Association, 1951.

GARCÍA SOLA, E. Diseño y Tecnología para viviendas de interés Social. [S.1.]: Facultad de Ingeniería de la Universidad Nacional del Nordeste/Dpto. de Física, Química y Geociencia,. 1974.

GONZALES, R. A.; PEREYRA, O.; SUIREZS, T. M.; ESKIVISKI, E. Estudio de las propiedades tecnológicas de las maderas de cinco especies forestales de interés industrial de Misiones. Yvyraretá, Eldorado, n. 11, 2003.

INSTITUTO ARGENTINO DE RACIONALIZACIÓN DE MATERIALES. Norma 9518. [Buenos Aires], 1962.

JUNTA DEL ACUERDO DE CARTAJENA. Manual del Grupo Andino para la Preservación de la Madera. [S.1], 1988. Proyecto de Promoción Industrial de la Madera para la Construcción (Prid-Madera).

LEONARDIS, J. Libro del árbol: esencias forestales no autóctonas cultivadas en la Argentina de aplicación ornamental y/o industrial. [S.1.]: Celulosa Argentina S.A. 1977. t. 3.

TINTO, J. Aporte del sector forestal a la construcción de viviendas. [S.l. s.n.],1978. 